\title{
A NOTE ON THE CONVOLUTION AND THE PRODUCT $D^{\prime}$ AND $S^{\prime}$
}

\author{
A. KAMIŃSKI and R. RUDNICKI \\ Institute of Mathemati."s \\ Polish Academy of Sciences \\ Nieczorka 8, 40-1) 13 Katowice \\ Poland
}

(Received November 27, 1989 and in revised form March 3, 1990)

ABSTRACT. Examples of tempered distributions are shown such that the convolution and product exist in $D^{\prime}$ and are tempered distributions, but they do not exist in $S^{\prime}$. On the other hand, there exist tempered distributions whose convolution or product exist in $D^{\prime}$, but are not tempered distributions.

KEY WORDS AND PHRASES. Tempered Distributions, Convolution and Product of Distributions.

1980 AMS SUBJECT CLASSIFICATION CODE. 46F10.

\section{INTRODUCTION.}

Various definitions of the convolution and the product have been considered in the space $D^{\prime}$ of distributions and in the space $S^{\prime}$ of tempered distributions, the duals to the spaces $D$ and $S$, respective1y (see Schwartz [1], pp. 21, 24, 233, 237).

In the case of the convolution $f^{*} g$ in $D^{\prime}$ (and $S^{\prime}$ resp.), most of the known definitions, e.g. those given by Schwartz [1] (p. 153), Chevalley [2], Shiraishi [3], Vladimirov [4] (p. 62), Dierolf and Volgt [5], are equivalent. For our purposes, we will consider the convolution in $D^{\prime}$ and in $S^{\prime}$ defined by means of so-called unitsequences, which are equivalent to the mentioned definitions for the respective classes of unit-sequences as shown in Kamiński [6]. By changing classes of unitsequences one can obtain, however more general definitions of the convolution in $D^{\prime}$ and in $S^{\prime}$.

The definitions of the product $f \cdot g$ in $D^{\prime}$ (in $S^{\prime}$ resp.) considered in the Literature are not equivalent. For instance, the definition for $D^{\prime}$ given by Hirata and Ogata [7] and by Mikusinski [8] by means of so-called delta-sequences depends essentially on the class of delta-sequences taken into consideration. Notice that given two tempered distributions it makes sense to consider the two operations of the convolution and product: in $D^{\prime}$ and in $S^{\prime}$. Therefore the following questions appear:

(I) Let $\mathrm{f}, \mathrm{g} \in S^{\prime}$. Suppose that $\mathrm{f}{ }_{\mathrm{g}}$ ( $\mathrm{f} \cdot \mathrm{g}$, resp.) exists in $D^{\prime}$. Does $\mathrm{f} \star_{\mathrm{g}}(\mathrm{f} \cdot \mathrm{g}$, resp.) belong to $S^{\prime}$ ?

(II) Let $f, g \in S^{\prime}$. Suppose that $f^{*} g$, ( $f \cdot g$, resp.) exists in $D^{\prime}$ and belongs to $S^{\prime}$. Does $\mathrm{f}^{*} \mathrm{~g}\left(\mathrm{f} \cdot \mathrm{g}\right.$, resp.) exist in $S^{\prime}$ ? 
The first question in the case of the convolution was posed by Shiraishi [3] and answered negatively by Kamírski [9] (see also Kamírsi [10]) and by Dierolf and Voigt [5] (see also Ortner and Wagner [11]). For the case of the product see Section 3.

The answer to the second question is also negative. A respective counter-example for the convolution in $\mathrm{R}^{2}$ was given by Wagner [12]. He also showed that the existence of the convolution $f * g$ in $D^{\prime}$ of homogeneous tempered distributions $f, g$ implies the existence of $\mathrm{f} * \mathrm{~g}$ in $\mathrm{D}^{\prime}$. Since Wagner's counter-example cannot be transferred into the one-dimensional case, we give a sultable example in Section 4. This counter-example can be automatically extended to $R^{d}$. Let us underline that in our example $f=g$ and $f$ is a real-valued distribution.

In the case of the product, a respective counter-example could be obtained from the example constructed for the convolution by means of the Fourier transform. This requires, however, careful considerations on relations between various definitions of the product of tempered distributions. Let us notice that the tempered distribution obtained in this way is complex-valued. Therefore we give in Section 5 a straightforward example with real-valued tempered distributions.

\section{DEFINITIONS.}

By $E$ we denote the class of all unit-sequences, i.e. sequences $\left(\eta_{n}\right)$ of functions of the class $D$ satisfying the conditions:

(i) $D^{k}\left(n_{n}-1\right) \rightarrow 0$ almost uniformly on $R^{d}$ for every multi-index $k=\left(k_{1}, \ldots, k_{d}\right)^{n} \varepsilon N_{0}^{d}$, where $N_{0}=N \cup\{0\}$.

(ii) For every $k \in N_{o}^{d}$ there is a constant $M_{k}>0$ such that

$$
\left|D^{k} n_{n}(x)\right|<M_{k}
$$

for a11 $x=\left(\xi_{1}, \ldots, \xi_{d}\right) \varepsilon R^{d}$ and $n \varepsilon N$.

By $E_{m}$ we denote the class of all model unit-sequences, i.e. sequences $\left(\eta_{n}\right)$ of the form

$$
\eta_{n}(x)=n\left(\alpha_{n} x\right), \quad x \in R^{d}, n \varepsilon N
$$

where $n$ is a function from such that $n(0)=0$ and $\left(\alpha_{n}\right)$ is a sequence of real numbers converging to 0 . Evidently, we have $E_{m} \subset E$.

Let $f, g \in D^{\prime}$ ( $f, g \in S^{\prime}$, resp.). We say that the convolution $f{ }^{\prime}$ exists in $D^{\prime}$ (in $S^{\prime}$, resp.) if the $11 \mathrm{mit}$

$$
\lim _{n \rightarrow \infty}\left(n_{n} f\right) \star\left(\tilde{n}_{n} g\right)
$$

exists in $D^{\prime}$ (in $S^{\prime}$, resp.) for all $\left(\eta_{n}\right),\left(\tilde{n}_{n}\right) \in E$. If

$$
\lim _{n \rightarrow \infty}\left(n_{n} f\right) \star\left(n_{n} g\right)
$$

exists for all $\left(\eta_{n}\right) \varepsilon E$, we say that the symmetric convolution $f * g$ exists in $D$ ' (in $S^{\prime}$ ). In both cases the limits do not depend on unit-sequences of the class E. In 
the above definitions the class $E$ can be replaced by $E_{m}$, but then we additionally assume that the limits $(2.1)$ and $(2.2)$ do not depend on model unit-sequences and $f{ }^{\prime} g$ is called the model (symmetric) convolution in $D^{\prime}$ (in $S^{\prime}$ ).

As shown in [6], the convolution in $D^{\prime}$ ( in $S^{\prime}$ ) as above is equivalent to the convolution in $D^{\prime}$ (in $S^{\prime}$ ) in the sense of the other known definitions mentioned in Section 1 .

If the convolution $f^{*} g$ exists in $D^{\prime}$ (in $S^{\prime}$ ), then the symmetric convolution $f *^{g}$ exists in $D^{\prime}$ (in $S^{\prime}$ ), but not conversely. If the (symmetric) convolution f*g exists in $D^{\prime}$ (in $S^{\prime}$ ), then the model (symmetric) convolution $\mathrm{f}^{\star} \mathrm{g}$ exists in $D^{\prime}$ (in $S^{\prime}$ ), but. not conversely.

By $\Delta_{0}$ we denote the class of all delta-sequences $\left(\delta_{n}\right)$, i.e. sequences of smooth functions satisfying the conditions:

(iii) there exists a sequence of positive numbers $\alpha_{n}, \alpha_{n}+0$ such that.

$\delta_{n}(x)=0$ for $|x|=\left(\xi_{1}^{2}+\ldots+\xi_{d}^{2}\right)^{1 / 2} \geqslant \alpha_{n}$ and $n \varepsilon N$

(iv) $\int \delta_{n}(t) d t=1$ for $n \in N$;

(v) there exists an $M>0$ such that

$$
\int\left|\delta_{n}(t)\right|<M \text { for } n \in N
$$

By $\Delta_{m}$ we denote the class of all model delta-sequences of the form

$$
\delta_{n}(x)=\alpha_{n}^{-d} \sigma\left(\alpha_{n}^{-1} x\right), \quad x \in R^{d}, n \varepsilon N
$$

where $\sigma \in \mathcal{D}, \int \sigma=1$ and $\left(\alpha_{\mathrm{n}}\right)$ is a sequence of real numbers converging to 0 . Evident $1 y, \Delta_{m} \subset \Delta_{o}$.

Let $\mathrm{f}, \mathrm{g} \varepsilon \mathrm{D}^{\prime}\left(\mathrm{f}, \mathrm{g} \varepsilon S^{\prime}\right.$, resp.). We say that the product $\mathrm{f} \cdot \mathrm{g}$ exists in $\mathcal{D}^{\prime}$ (in $S^{\prime}$, resp.) if the limit

$$
\lim _{n \rightarrow \infty}\left(f * \delta_{n}\right) \cdot\left(g * \tilde{\delta}_{n}\right)
$$

exists in $D^{\prime}$ (in $S^{\prime}$, resp. ) for all $\left(\delta_{n}\right),\left(\tilde{\delta}_{n}\right) \in \Delta_{0}$. If

$$
\lim _{n+\infty}\left(f * \delta_{n}\right) \cdot\left(g * \delta_{n}\right)
$$

exists for all $\left(\delta_{n}\right) \in \Delta_{0}$, we say that the symmetric product exists in $D^{\prime}$ (in $S^{\prime}$ ). If $\Delta_{0}$ is replaced by $\Delta_{m}$, f.g is called the model (symmetric) product in $D^{\prime}$ (in $S^{\prime}$ ). one can show that the limirs (2.3) and $(2.4)$ do not depend on delta-sequences from $\Delta_{0}$. We assume this for the class $\Delta_{m}$.

For these and other definitions of the product in $D^{\prime}$ and in $S^{\prime}$ and their equivalence see Mikusiński [8 and [13], Hirata and Ogata [7], Shiraishi and Itano [14], Antosik, Mikusiński and Sikorski [15] (p. 242), Kamiŕski [6], Oberguggenberger [16], and Wawak [17, 18]. Note that if the product f.g exists in $\mathcal{O}^{\prime}$ (in $S^{\prime}$ ), then the symnetric product $f . g$ exists in $D^{\prime}$ (in $S^{\prime}$ ), but not conversely. If the 
(symmetric) product f.g exist.s in $D$ (in $S^{\prime}$ ), then the model (symmetric) product f.g exists in $D^{\prime}$ (in $S^{\circ}$ ), but not conversely.

3. QUESTION (I) FOR PRODUCT.

It is very easy to give the negative answer lo the question (I) for the product in $R^{l}$ in case complex-valued distributions (functions) are admitted. Namely, given an arbitrary real-valued continuous function $F$ on $R^{l}$ (in particular, $F$ can be chosen so that $\mathrm{F}\left(\mathrm{S}^{\prime}\right)$ put

$$
\begin{aligned}
& G(x)=\exp \left[i \int_{0}^{x} F(t) d t\right] \\
& f_{1}(x)=G^{\prime}(x)=1 G(x) F(x)
\end{aligned}
$$

and

$$
f_{2}(x)=-i G^{-1}(x)
$$

for $x \in R^{1}$. Then $f_{1}, f_{2}$ are continuous complex-valued functions on $R^{l}$ such that $\mathrm{f}_{1} \cdot \mathrm{f}_{2}=\mathrm{F}$ in the usual sense, so the more in $D^{\prime}$, and $\mathrm{f}_{1}, \mathrm{f}_{2} \varepsilon S^{\prime}$, because $|\mathrm{G}(\mathrm{x})|=1$.

We are going to show that there exists also a real-valued smooth function $\phi$ which belongs to $S^{\prime}$ such that the square $\phi^{2}$ (which also exists in the sense of the product in $\left.D^{\prime}\right)$ does not belong to $S^{\prime}$. First denote $I_{j}=\left[j-3^{-j}, j+3^{-j}\right], J_{j}=\left[j-2.3^{-j}\right.$, $j+2.3^{-j}$ ] for $j \varepsilon N$. Let $\phi$ be a smooth function such that

$$
\begin{array}{ll}
\phi(x)=2^{j}, & x \in I_{j}, \\
0<\phi(x)<2^{j}, & x \in J_{i},
\end{array}
$$

and

$$
\phi(x)=0, \quad x \dot{\xi} ?_{j=1}^{\infty} J_{j}
$$

for $j \varepsilon N . \quad B y(3.1)$ we have

$$
\int_{0}^{\infty} \phi(t) d t<\sum_{j=1}^{\infty} 2^{j}\left|J_{j}\right|=4 \sum_{j=1}^{\infty}(2 / 3)^{j}<\infty,
$$

so $\phi \varepsilon S^{\prime}$. Since $\phi \star \delta_{n}+\phi$ almost uniformly for any $\left(\delta_{n}\right) \varepsilon \Delta_{0}$, the product $\phi \cdot \phi$ exists in $D^{\prime}$ and coincides with the usual product $\phi^{2}$ of functions. Taking $\omega(x)=\exp (-x / 4)$, we have

$$
\int_{0}^{\infty} \phi^{2}(x) \omega(x) d x \geq 2 \alpha \sum_{j=1}^{\infty}(4 \alpha / 3)^{j}=\infty,
$$

where $\alpha=\exp (-1 / 4)$. Consequent1y, $\phi^{2} \varepsilon S^{\prime}$.

Another example, given explicitly by $\phi(x)=e^{x} \cos e^{x}$ but based on similar calculations, was communicated to us by $P$. Wagner.

4. QUESTION (II) FOR CONVOLUTION.

Now, we shall construct an example of a distribution $f \varepsilon S^{\prime}$ such that the convolution square $f^{\star} f$ exist in $D^{\prime}$ and is of the class $S^{\prime}$, but does not exist in $S^{\prime}$. 
Let $\mathrm{f}=\mathrm{F}_{1}+\mathrm{f}_{2}$ with

$$
f_{1}(x)=\sum_{j=2}^{\infty}(-1)^{j} 2^{\left[\log _{2} j\right]} \delta\left(x-2^{j}\right)
$$

and

$$
f_{2}(x)=\sum_{j=2}^{\infty} \delta\left(x+2^{j}-\left[\log _{2} j\right]\right)
$$

where the symbol $[\mathrm{x}]$ means the integer part of $x$. It is clear that $\mathrm{f}_{1}, \mathrm{f}_{2}, \mathrm{f} \varepsilon S^{\prime}$. Note that

$$
\begin{aligned}
& A=\operatorname{supp} f_{1}=\left\{2^{k}: k \varepsilon N \backslash\{1\}\right\} \\
& B=\operatorname{supp} f_{2}=\left\{-2^{k}+\left[\log _{2} k\right]: k \varepsilon N \backslash\{1\}\right\} .
\end{aligned}
$$

Each of the sets supp $f_{1}$ and supp $f_{2}$ is bounded from one side, so it is polynomially compatible in the sense of Kaminski [10]. Therefore the convolutions $\mathrm{f}_{1} * \mathrm{f}_{1}$ and $\mathrm{f}_{2} * \mathrm{f}_{2}$ exists in $S^{\prime}$ (cf. [9] and [10]). It suffices to show that the convolution $\mathrm{f}_{1} * \mathrm{f}_{2}=\mathrm{f}_{2} * \mathrm{f}_{1}$ exists in $D^{\prime}$ and does not exist in $S^{\prime}$. To prove that $\mathrm{F}_{1}$ * $\mathrm{F}_{2}$ exists in $D^{\prime} i t$ is enough to verify that for every bounded interval $I$ in $R^{l}$ the set

$$
\{(x, y): x \in A, y \varepsilon B, x+y \varepsilon I\}
$$

is bounded in $R^{2}$. But if

$$
\left|2^{j}-2^{k}+\left[\log _{2} k\right]\right|<m
$$

for some $m \varepsilon N$, then we have

and

$$
\max (j, k)<2+\log _{2} m \quad \text { for } j \neq k
$$

$$
j, k<2^{m+1} \quad \text { for } j=k \text {, }
$$

which proves the required property. In addition, we have

$$
\mathrm{f}_{1} * \mathrm{f}_{2}=\sum_{j=2}^{\infty} \sum_{k=2}^{\infty}(-1)^{j} 2^{\left[\log _{2} j\right]} \delta\left(x-2^{j}+2^{k}-\left[\log 2^{k]}\right)\right. \text {. }
$$

In order to prove that $\mathrm{f}_{1} * \mathrm{f}_{2} \varepsilon S^{\prime}, \mathrm{fix} m \varepsilon \mathrm{z}$ and consider the pairs $(\mathrm{j}, \mathrm{k})$ for which

$$
2^{j}-2^{k}+[\log k]=m \text {. }
$$

If $j=k$ then

$$
f=k \varepsilon\left\{2^{m}, 2^{m}+1, \ldots, 2^{m+1}-1\right\}
$$

If $j \neq k$ then there exists at most one pair $(j, k)$ such that (4.1) holds and $|m|>2^{j-1}>j$. Setting $J=\left\{j \varepsilon N: 2^{m}<j<2^{m+1}-1\right\}$, we obtain 


$$
\sum_{j \in J}(-1)^{j} 2^{m} \delta(x-m)=0
$$

Hence

$$
\left(f_{1} * f_{2}\right)(x)=\sum_{m \varepsilon Z} a_{m} \delta(x-m),
$$

where $a_{m}=(-1)^{j} 2^{\left[\log _{2} j\right]}$ if there exists a pair $(j, k)$ such that $(4.1)$ holds and $a_{m}=0$ otherwise. In both cases $\left|a_{m}\right|<j<m$, which implies that $f_{1} * f_{2} \varepsilon S^{\prime}$. Now, let $\eta$ be a real-valued function of the class $D$ such that $n(x)=1$ for $|x|<1$ and $n(x)=0$ for $|x|>3 / 2$. Setting $\eta_{n}(x)=n(x / n)$ we have, for $n=2^{2^{m}}$.

$$
\eta_{n}(x) f_{1}(x)=\sum_{j=2}^{2^{m}}(-1)^{j} 2^{\left[\log _{2} j\right]} \delta\left(x-2^{j}\right)
$$

and

$$
\eta_{n}(x) f_{2}(x)=\sum_{k=2}^{2^{m}} \delta\left(x+2^{k}-\left[\log _{2} k\right]\right) .
$$

From the last formulae it follows that the distribution $\left(n_{n} f_{1}\right) *\left(n_{n} f_{2}\right)$ can be expressed as a sum of two distributions $g_{m}=\Sigma_{1} \Phi_{j j}$ and $h_{m}=\Sigma_{2} \Phi_{j k}$, where

$$
\Phi_{j k}=(-1)^{j} 2^{\left[\log _{2} j\right]} \delta\left(x-2^{j}+2^{k}-\left[\log _{2} k\right]\right)
$$

and $\Sigma_{1}$ denotes the summation over all $j$ with $2<j<2^{m}$ and $\Sigma_{2}$ denotes the summation over all $j$ and $k$ with $2<j, k<2^{m}, j \neq k$. Setting $h=\Sigma_{3} \Phi_{j k}$, where $\Sigma_{3}$ denotes the summation over all $j, k>2$ with $j \neq k$, it is easy to check that

$$
\lim _{m \rightarrow \infty}\left\langle h_{m}, \phi\right\rangle=\langle h, \phi\rangle
$$

for all $\phi \varepsilon S$. On the other hand, taking $\phi \varepsilon S$ such that $\phi(x)=(2 / 3)^{x}$ for $x>0$, we have, in view of (4.2),

$$
\begin{aligned}
\left\langle g_{m}, \phi\right\rangle & =\left\langle 2^{m} \delta(x-k), \phi(x)\right\rangle \\
& =2^{m} \phi(k)=(4 / 3)^{m}+\infty
\end{aligned}
$$

as $m \rightarrow \infty_{0}$ Therefore the convolutions $\mathrm{f}_{1} * \mathrm{f}_{2}=\mathrm{f}_{2} * \mathrm{f}_{1}$ do not exist in $S^{\prime}$ and this means that $f^{\star} f$ does not exist in $S^{\prime}$.

REMARK 4.1. Note that in the construction above $f \star_{f}$ does not exist in $S^{\prime}$ even in the sense of the symmetric model convolution. Another example of a distribution $f \varepsilon S^{\prime}$ such that the convolution $f^{*} f$ exists in $D^{\prime}$ and does not exist in $S^{\prime}$ can be obtained by taking $\mathrm{f}=\mathrm{g}_{1}+\mathrm{g}_{2}$ with

$$
g_{1}(x)=\sum_{k=2}^{\infty}(-1)^{k} \delta\left(x-2^{k}\right), g_{2}(x)=\sum_{k=2}^{\infty} \delta\left(x+2^{k}-\left[\log _{2} k\right]\right) .
$$

In this example $f * f$ exists in $S^{\prime}$ in the sense of the limit (2.3) for all unitsequences $\left(n_{n}\right)$, $\left(\tilde{n}_{n}\right)$ with a common1y bounded variation. In paricular, the model convolution $f * f$ exists in $S^{\prime}$, in contrast to the example presented before. 
REMARK 4.2. Put $F=\mathrm{f} * \omega$, where $\mathrm{f}$ is the distribution constructed in the example above and $\omega$ is a function of the class $D$. Then $F$ is a slowly increasing (i.e. bounded by a polynomial) smooth function such that the convolution F*F exists in $D^{\prime}$ and represents a slowly increasing smooth function while the (model symmetric) convolution $F^{\star} F$ does not exist in $S^{\prime}$. The example can also be modified in such a way that $f$ is a distribution (even a smooth function) from an arbitrary Gelfand-Shilov space $K^{\prime}\left(M_{p}\right)$ with the property: the convolution $f \star_{f}$ exists in $D^{\prime}$ and is a distribution (smooth function) of the class $K^{\prime}\left(M_{p}\right)$, but $f * f$ does not exist in $K^{\prime}\left(M_{p}\right)$.

\section{QUESTION (II) FOR PRODUCT.}

Now we are going to give a simple example of two tempered distributions $f, g$ on $R^{1}$ such that the product f.g exists in $D^{\prime}$ and belongs to $S^{\prime}$, but f.g does not exist in $S^{\prime}$. Let

$$
f(x)=\sum_{k=1}^{\infty} \delta(x-k), \quad g(x)=\sum_{k=1}^{\infty} \delta\left(x-k-2^{-k}\right) .
$$

Obviously, $\mathrm{f}, \mathrm{g} \varepsilon S^{\prime}$. Since the product $\delta(\mathrm{x}-\mathrm{a}) \delta(\mathrm{x}-\mathrm{b})$ exists in $D^{\prime}$ and equals 0 for $a \neq b$, it follows that the product $f \cdot g$ exists in $D^{\prime}$ and $f \cdot g=0$. To prove that the symmetric model product $\mathrm{f} . \mathrm{g}$ does not exist in $D^{\prime}$ define

$$
\delta_{n}(x)=n \omega(n x)
$$

where $\omega$ is a non-negative function of the class $D$ such that

$$
\omega(x)=1 \quad \text { for } x \varepsilon[-1 / 4,1 / 4]
$$

and

$$
\int \omega(x) d x=1
$$

Moreover, consider a positive function $\phi$ of the class $S$ such that $\phi(x)=(2 / 3)^{x}$ for $x$ >. Then we have

$$
\begin{gathered}
\left\langle\left(f * \delta_{n}\right) \cdot\left(g * \delta_{n}\right), \phi\right\rangle>\sum_{m=1}^{\infty} \int \delta_{n}(x-m) \delta_{n}\left(x-m-2^{-m}\right) \phi(x) d x \\
\quad>\int \delta_{n}(x-j) \delta_{n}\left(x-j-2^{-j}\right) \phi(x) d x>2^{j-4}(2 / 3)^{j+1},
\end{gathered}
$$

where $j=n+2$. Since $2^{j-4}(2 / 3)^{j+1}+\infty$ as $j+\infty$, the symmetric model product f.g does not exist in $S$.

Fina1ly, let us pose the following open question:

PROBLEM. Does there exist a real-valued tempered distribution $f$ such that the product $\mathrm{f} . \mathrm{g}$ exists in $D^{\prime}$ and belongs to $S^{\prime}$, but the (symmetric model) product f.f does not exist in $S^{\prime}$ ? 
1. SCHWARTZ, L. Theorie des Distrlbutions, Hermann, Paris, 1966.

2. CHEvalley, C. Theory of Distributions, Lectures at Columbia Univ., 1950-51.

3. ShIRAISHI, R. On the Definition of Convolutions for Distributions, J. Sci. Hi roshima Univ. Ser. A. 23(1) (1959), 19-32.

4. VLADIMIROV, V.S. Generalized Functions in Mathematical Physics, Mir, Moscow,

5. DIEROLF, P. and VOIGT, J. Convolution and $S^{\prime}$-convolution of Distributions, Collect. Math. 29(3), (1978), 185-196.

6. KAMINSKI, A. Convolution, Product and Fourier Transform of Distributions, Studia Math. 74 (1982), 83-96.

7. HIRATA, Y. and OGATA, H. On the Exchange Formula for Distributions, J. Sc1. Hiroshima Univ. Ser. A 22 (1958), 147-152.

8. MIKUSIŃSKI, J. Criteria of the Existence and of the Associativity of the Product of Distributions, Studia Math. 21 (1962), 253-259.

9. KAMIŃSKI, A. Calkowanie 1 operacje nieregularne, Ph. D. Dissertation, Institute of Mathematics, Polish Academy of Sciences, Warsaw, 1975.

10. KAMIŃSKI, A. On Convolutions, Products and Fourier Transforms of Distributions, Bu11. Acad. Polon. Sci. Sef. Sc1. Math. Astronom. Phys. 25 $(1977), 369-374$.

11. ORTNER, N. and WAGNER, P. Sur quelques propriétés des espaces $D^{\prime}$ de Laurent
Schwartz, Bol1. Un. Mat. Ita1. (6) 2-B (1983), 353-376.

12. WAGNER, P. Zur Faltung von Distributionen, Math. Ann. 276 (1987), 467-485.

13. MIKUSINSKI, J. On the Square of the Dirac Delta-distibution, Bul1. Acad. Polon. Sc1. Ser. Sci. Math. Astornom. Phys. 8 (1960), 681-683.

14. SHIRAISHI, R. and ITANO, M. On the Multiplicative Products of Distributions, J. Sci. Hi roshima Univ. Ser. A 28 (1964), 223-235.

15. ANTOSIK, P., MIKUSINSKI, J. and SIKORSKI, R. Theory of Distributions. The Sequential Approach, PWN-E1sevier, Warszawa-Amsterdam, 1973.

16. OBERGUGgenberger, M. Products of Distributions, J. Reine Angew. Math. 365 (1986), 1-11.

17. WAWAK, R. Improper Integrals of Distributions, Studia Math. 86 (1987), 205-220.

18. WAWAK, R. On the Colombeau Product of Distributions, Proc. of the Conf. on Generalized Functions and Convergence, Katowlce 1988 (to appear). 


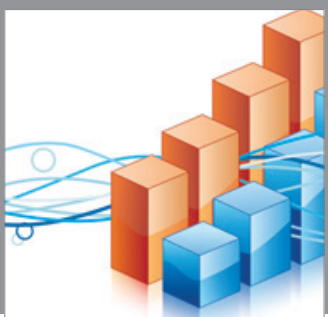

Advances in

Operations Research

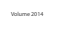

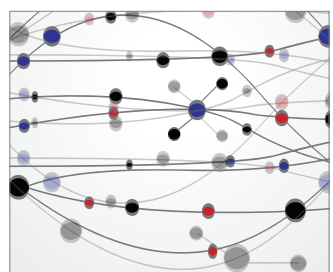

\section{The Scientific} World Journal
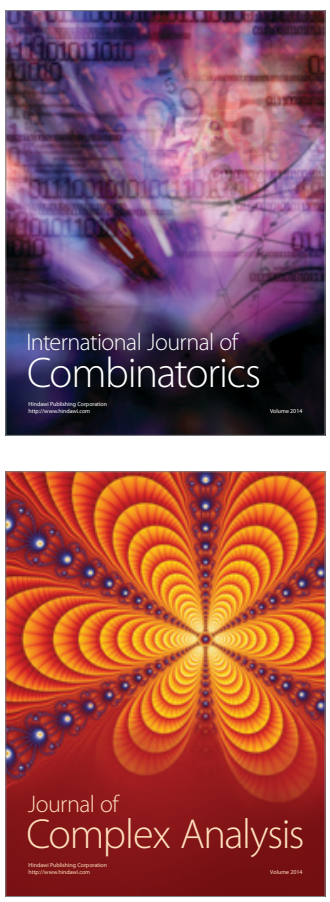

International Journal of

Mathematics and

Mathematical

Sciences
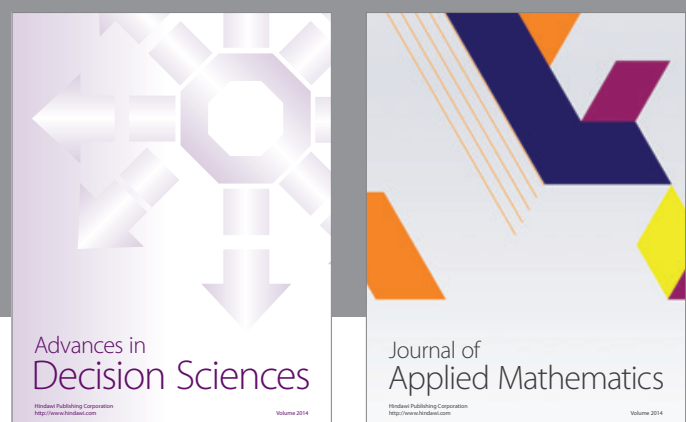

Journal of

Applied Mathematics
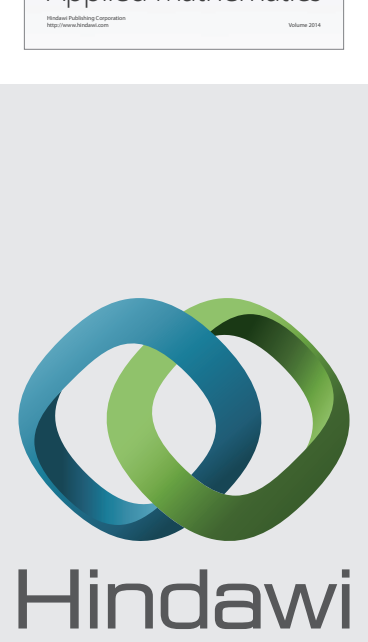

Submit your manuscripts at http://www.hindawi.com
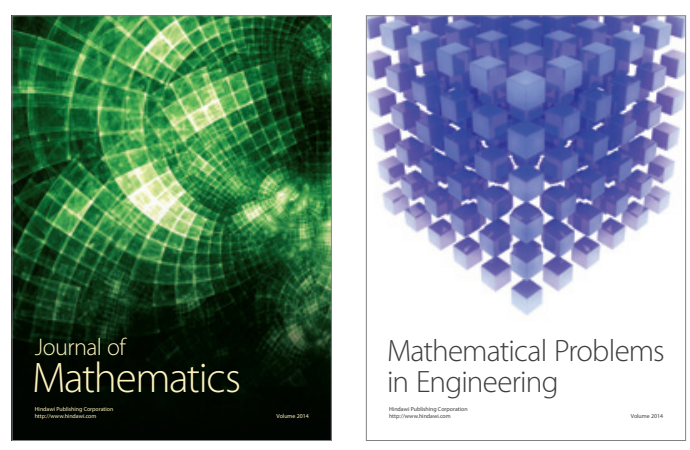

Mathematical Problems in Engineering
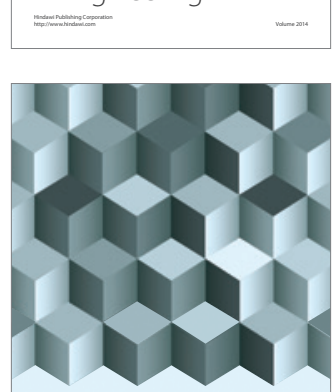

Journal of

Function Spaces
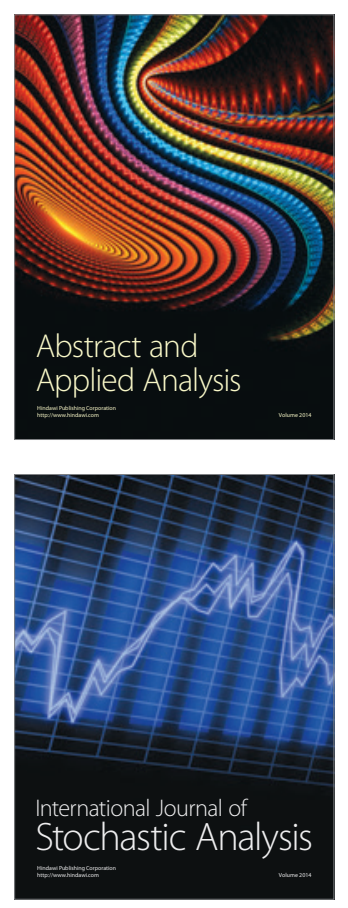

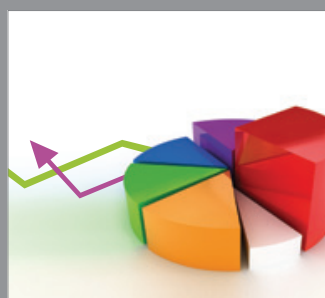

ournal of

Probability and Statistics

Promensencen
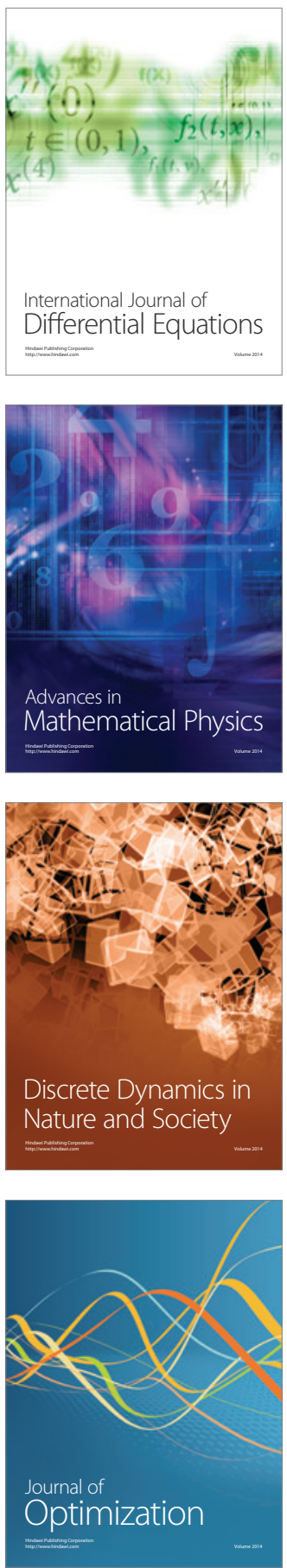